\title{
Fekete - Szego Problem for Sakaguchi kind of Functions Related to Shell - like Curves Connected with Fibonacci Numbers
}

\section{P.Lokesh, B.Srutha keerthi}

\begin{abstract}
In this paper, it is attempted to introduce and investigate new subclasses of Sakaguchi kind of functions related to shell - likes curves connected with Fibonacci numbers. Furthermore, the estimates of first two coefficients of functions in these classes are obtained. Fekete - Szego inequalities for these function classes are also determined.
\end{abstract}

Keywords : Fibonacci numbers, Sakaguchi kind of functions, Fekete - Szego inequalities.

\section{INTRODUCTION}

Let $\Omega=\{z:|z|<1\}$ denote the unit disc on the complex plane. The class of all analytic functions of the form

$f(z)=z+\sum_{n=1}^{\infty} a_{n} z^{n}$

in the open unit disc $\Omega$ with normalization $f(0)=f^{\prime}(0)-1=0$ is denoted by $A$ and class $S \subset A$ is the class which consists of univalent functions in $\Omega$.

The koebe one quarter theorem [3] ensures that the image of $\Omega$ under every univalent function $f \in A$ contains a disk of radius $1 / 4$. Thus every univalent function $f \in A_{\text {has an inverse }} f^{-1}$ satisfying

$$
\begin{gathered}
f^{-1}(f(z))=z, \quad(z \in \Omega) \\
f\left(f^{-1}(w)\right)=w\left(|w|<r_{0}(f), r_{0}(f) \geq \frac{1}{4}\right) .
\end{gathered}
$$

and

A function $f \in A$ is said to be bi-univalent on $\Omega$ if both $f$ and $f^{-1}$ are univalent in $\Omega$. Let $\Sigma$ denote the class of bi univalent functions as defined in the unit disk $\Omega$. Since $f \in \Sigma$ has the Maclaurian Series given by (1), a computation shows that its inverse $g=f^{-1}$ has the

\section{Revised Manuscript Received on July 08, 2019.}

First Author Name, his/her department, Name of the affiliated College or University/Industry, City, Country. Email: xyz1@ @blueeyesintlligence.org

Second Author Name, department, Name of the affiliated College or University/Industry, City, Country. Email: xyz2@blueeyesintlligence.org

Third Author Name, department, Name of the affiliated College or University/Industry, City, Country. Email: xyz3@blueeyesintlligence.org expansion

$$
g(w)=f^{-1}(w)=w-a_{2} w^{2}+\left(2 a_{2}^{2}-a_{3}\right)+\ldots . .
$$

One can see a short history and examples of function in the class $\Sigma$ in [13]. Several authors have introduced and investigated subclasses of bi - univalent functions and obtained bounds for the initial coefficients (see [1, $2,8,13,14,15]$ ).

An analytic function $f$ is subordinate to an analytic function $F$ in $\Omega$, written as $f \pi F(z \in \Omega)$, provided there is an analytic function $w$ defined on $\Omega$ with $w(0)=0$ and $|w(z)|<1$ satisfying $f(z)=F(w(z))$. It follows from Schwarz Lemma that

$$
f(z) \pi F(z) \Leftrightarrow f(0)=F(0)
$$

and $f(\Omega) \subset F(\Omega), z \in \Omega$

(for details see [3,7]. The important subclasses of $S$ in geometric function theory such that if $f \in A$ are recalled and

$$
\frac{z f(z)}{f(z)} \pi p(z) \text { and } 1+\frac{z f^{\prime \prime}(z)}{f^{\prime}(z)} \pi p(z)
$$

where $p(z)=\frac{1+z}{1-z}$, then it is said that $f$ is star like and convex, respectively. These functions form known classes denoted by $S^{*}$ and $C$, respectively. Recently, in [12], Sokol introduced the class $S L$ of Shell-like functions on the set of functions $f \in A$ which is described in the following definitions:

Definition 1.1. The function $f \in A$ belongs to the class $S L_{\text {if it satisfies the condition that }}$ $\frac{z f^{\prime}(z)}{f(z)} \pi \tilde{p}(z)$

with

$$
\tilde{p}(z)=\frac{1+\tau^{2} z^{2}}{1-\tau z-\tau^{2} z^{2}}
$$

where 
Fekete - Szego Problem for Sakaguchi kind of Functions Related to Shell - like Curves Connected with Fibonacci numbers

$\tau=\frac{(1-\sqrt{5})}{2} \approx-0.618$

It should be observed $S L$ is a subclass of the star like functions $S^{*}$.

Later, Dziok et al. in [4] and [5] and Ozlem Guney et al. [9] defined and introduced various subclasses of bi-univalent function related to a shell - like curve connected with Fibonacci numbers, respectively.

Definition 1.2. The function $f \in A$ belongs to the class $K S L$ of convex shell - like functions if it satisfies the condition that

$$
\begin{gathered}
1+\frac{z f^{\prime \prime}(z)}{f^{\prime}(z)} \pi \tilde{p}(z)=\frac{1+\tau^{2} z^{2}}{1-\tau z-\tau^{2} z^{2}} \\
\text { with } \tau=\frac{(1-\sqrt{5})}{2} \approx-0.618
\end{gathered}
$$

The function $\tilde{p}$ is not univalent in $\Omega$, but it is univalent in the $\operatorname{disc}|z|<\frac{(3-\sqrt{5})}{2} \approx 0.38$ $\tilde{p}(0)=\tilde{p}(-1 / 2 \tau)=1$ and $\tilde{p}\left(e^{\mu i \arccos (1 / 4)}\right)=\frac{\sqrt{5}}{5}$, and it may also be noticed that $\frac{1}{|\tau|}=\frac{|\tau|}{1-|\tau|}$

which shows that the number $|\tau|$ divides [0,1] such that it fulfills the golden section. The image of the unit circle $|z|=1$ under $\tilde{p}$ is a curve described by the equation given by

$$
(10 x-\sqrt{5}) y^{2}=(\sqrt{5}-2 x)(\sqrt{5 x}-1)^{2},
$$

which is translated and revolved trisectrix of Maclaurin. The curve $\tilde{p}\left(r e^{i t}\right)$ is a closed curve without any loops for $0<r \leq r_{0}=\frac{(3-\sqrt{5})}{2} \approx 0.38$ For $r_{0}<r<1$, it has a loop and for $r=1$, it has a vertical asymptote. Since $\tau$ satisfies the equation $\tau^{2}=1+\tau$, this expression can be used to obtain higher powers $\tau^{n}$ as a linear function of lower powers, which in turn can be decomposed all the way down to a linear combination of $\tau$ and 1 . The resulting recurrence relationships yield Fibonacci numbers $u_{n}$ :

$$
\tau^{n}=u_{n} \tau+u_{n-1}
$$

In [11], taking $\tau z=t$, Raina and Sokol showed that

$$
\tilde{p}(z)=\frac{1+\tau^{2} z^{2}}{1-\tau z-\tau^{2} z^{2}}=\left(t+\frac{1}{t}\right) \frac{1}{1-t-t^{2}}
$$

$$
\begin{aligned}
& =\frac{1}{\sqrt{5}}\left(t+\frac{1}{t}\right)\left(\frac{1}{1-(1-\tau) t}-\frac{1}{1-\tau t}\right) \\
& =\left(t+\frac{1}{t}\right) \sum_{n=1}^{\infty} \frac{(1-\tau)^{n}-\tau^{n}}{\sqrt{5}} t^{n}
\end{aligned}
$$

$=\left(t+\frac{1}{t}\right) \sum_{n=1}^{\infty} u_{n} t^{n}=1+\sum_{n=1}^{\infty}\left(u_{n-1}+u_{n+1}\right) \tau^{n} z^{n}$,

where

$$
u_{n}=\frac{(1-\tau)^{n}-\tau^{n}}{\sqrt{5}}, \tau=\frac{1-\sqrt{5}}{2}(n=1,2, \ldots .)
$$

This shows that the relevant connection of $\tilde{p}$ with the sequence of Fibonacci numbers $u_{n}$, such that $u_{0}=0, u_{1}=1, u_{n+2}=u_{n}+u_{n+1}$ for $\mathrm{n}=0,1,2, \ldots$. And they got

$$
\begin{aligned}
& \tilde{p}(z)=1+\sum_{n=1}^{\infty} \tilde{p}_{z} z^{n}=1+\left(u_{0}+u_{2}\right) \pi+\left(u_{1}+u_{3}\right) z^{2} z^{2}+\sum_{n=3}^{\infty}\left(u_{n-3}+u_{n-2}+u_{n-1}+u_{n}\right) z^{n} z^{n} \\
&=1+\tau z+3 \tau^{2} z^{2}+4 \tau^{3} z^{3}+7 \tau^{4} z^{4}+11 \tau^{5} z^{5}+\ldots
\end{aligned}
$$

Let $p(\beta), 0 \leq \beta<1$, denote the class of analytic functions $p$ in $\Omega$ with $p(0)=1$, and $\operatorname{Re}\{p(z)\}>\beta$. Especially, $p$ instead of $p(0)$ is used.

Theorem 1.3. [5] The function

$$
\tilde{p}(z)=\frac{1+\tau^{2} z^{2}}{1-\tau z-\tau^{2} z^{2}}
$$

belongs to the class $p(\beta)_{\text {with }} \beta=\frac{\sqrt{5}}{10} \approx 0.2236$.

Now the following lemma is given to prove the theorem.

$$
\begin{gathered}
p(z)=1+c_{1} z+c_{2} z^{2}+\ldots \text { then } \\
\left|c_{n}\right| \leq 2 \text {, for } n \geq 1 \text {. (6) }
\end{gathered}
$$$$
\text { Lemma 1.4. [10] Let } p \in P \text { with }
$$

In this present work, two subclasses of Sakaguchi kind of $\Sigma$ associated with shell - like functions connected with Fibonacci numbers are introduced to obtain the initial Taylor coefficients $\left|a_{2}\right|$ and $\left|a_{3}\right|$ for these function classes. Also, bounds for the Fekete - Szego functional $\left|a_{3}-\mu a_{2}^{2}\right|$ for each subclass are also given. 


\section{BI- UNIVALENT FunCtion Class $S L M_{\lambda, t, \Sigma}(\tilde{p}(z))$}

In this section, a new subclass of Sakaguchi kind of $\Sigma$ is associated with shell - like functions connected with Fibonacci numbers in order to obtain the initial Taylor coefficients $\left|a_{2}\right|$ and $\left|a_{3}\right|$ for the function class of subordination.

Firstly, let $p(z)=1+p_{1} z+p_{2} z^{2}+\ldots \quad$ and $p \pi \tilde{p}$. Then there exists an analytic functions $u$ such that $|u(z)|<1$ in $\Omega$ and $p(z)=\tilde{p}(u(z))$. Therefore the function

$$
h(z)=\frac{1+u(z)}{1-u(z)}=1+c_{1} z+c_{2} z^{2}+\ldots .
$$

is in the class $p(0)$. It follows that

$$
u(z)=\frac{c_{1} z}{2}+\left(c_{2}-\frac{c_{1}^{2}}{2}\right) \frac{z^{2}}{2}+\left(c_{3}-c_{1} c_{2}-\frac{c_{1}^{3}}{4}\right) \frac{z^{3}}{2}+\ldots . .
$$

and

$$
\begin{aligned}
& \tilde{p}(u(z))=1+\tilde{p}_{1}\left(\frac{c_{1} z}{2}+\left(c_{2}-\frac{c_{1}^{2}}{2}\right) \frac{z^{2}}{2}+\left(c_{3}-c_{1} c_{2}-\frac{c_{1}^{3}}{4}\right) \frac{z^{3}}{2}+\ldots\right\} \\
& +\tilde{p}_{2}\left\{\frac{c_{1} z}{2}+\left(c_{2}-\frac{c_{1}^{2}}{2}\right) \frac{z^{2}}{2}+\left(c_{3}-c_{1} c_{2}-\frac{c_{1}^{3}}{4}\right) \frac{z^{3}}{2}+\ldots .\right\} \\
& +\tilde{p}_{3}\left\{\frac{c_{1} z}{2}+\left(c_{2}-\frac{c_{1}^{2}}{2}\right) \frac{z^{2}}{2}+\left(c_{3}-c_{1} c_{2}-\frac{c_{1}^{3}}{4}\right) \frac{z^{3}}{2}+\ldots\right\}^{3}+\ldots . . \\
& =1+\frac{\tilde{p}_{1} c_{1} z}{2}+\left\{\frac{1}{2}\left(c_{2}-\frac{c_{1}^{2}}{2}\right) \tilde{p}_{1}+\frac{c_{1}^{2}}{4} \tilde{p}_{2}\right\} z^{2}+ \\
& \left\{\frac{1}{2}\left(c_{3}-c_{1} c_{2}+\frac{c_{1}^{2}}{4}\right) \tilde{p}_{1}+\frac{1}{2} c_{1}\left(c_{2}-\frac{c_{1}^{2}}{2}\right) \tilde{p}_{2}+\frac{c_{1}^{3}}{8} \tilde{p}_{3}\right\} z^{3}+\ldots \ldots . .
\end{aligned}
$$

And similarly, there exists an analytic function $v$ such that $|v(w)|<1$ in $\Omega$ and $p(w)=\tilde{p}(v(w))$. Therefore, the function

$$
K(w)=\frac{1+v(w)}{1-v(w)}=1+d_{1} w+d_{2} w^{2}+\ldots
$$

is in the class $P(0)$. It shows that

$$
v(w)=\frac{d_{1} w}{2}+\left(d_{2}-\frac{d_{1}^{2}}{2}\right) \frac{w^{2}}{2}+\left(d_{3}-d_{1} d_{2}-\frac{d_{1}^{2}}{4}\right) \frac{w^{3}}{2}+\ldots \ldots
$$

And

$$
\begin{aligned}
& \tilde{p}(v(w))=1+\frac{\tilde{p}_{1} d_{1} w}{2}+\left\{\frac{1}{2}\left(d_{2}-\frac{d_{1}^{2}}{2}\right) \tilde{p}_{1}+\frac{d_{1}^{2}}{4} \tilde{p}_{2}\right\} w^{2}+ \\
& \left\{\frac{1}{2}\left(d_{3}-d_{1} d_{2}+\frac{d_{1}^{2}}{4}\right) \tilde{p}_{1}+\frac{1}{2} d_{1}\left(d_{2}-\frac{d_{1}^{2}}{2}\right) \tilde{p}_{2}+\frac{d_{1}^{3}}{8} \tilde{p}_{3}\right\} w^{3}+\ldots \ldots . .
\end{aligned}
$$

Definition 2.1. For $0 \leq \lambda \leq 1,|t| \leq 1$ but $t \neq 1$ a function $f \in A$ of the form (1) is said to be in the class $S L M_{\lambda, t, \Sigma}(\tilde{p}(z))$ if the following subordination hold: $\frac{(1-t)\left(\lambda z^{3} f^{\prime \prime \prime}(z)+(1+2 \lambda) z^{2} f^{\prime \prime}(z)+z f^{\prime}(z)\right)}{\lambda z^{2}\left(f^{\prime \prime}(z)-t^{2} f^{\prime \prime}(t z)\right)+z\left(f^{\prime}(z)-t f^{\prime}(t z)\right)} \pi \tilde{p}(z)=\frac{1+\tau^{2} z^{2}}{1-\tau z-\tau^{2} z^{2}}$

and

$\frac{(1-t)\left(\lambda w^{3} g^{\prime \prime \prime}(w)+(1+2 \lambda) w^{2} g^{\prime \prime}(w)+w g(w)\right)}{\lambda w^{2}\left(g^{\prime \prime}(w)-t^{2} g^{\prime \prime}(t w)\right)+w\left(g^{\prime}(w)-t g^{\prime}(t w)\right)} \pi \tilde{p}(w)=\frac{1+\tau^{2} w^{2}}{1-\tau w-\tau^{2} w^{2}}$

Where

$$
\tau=\frac{(1-\sqrt{5})}{2} \approx-0618
$$

is given by (2).

In the following theorem, an attempt has been made to determine the initial Taylor coefficients $\left|a_{2}\right|$ and $\left|a_{3}\right|$ for the function $S L M_{\lambda, t, \Sigma}(\tilde{p}(z))$. Fekete - Szego functional $\left|a_{3}-\mu a_{2}^{2}\right|$ for this subclass is also obtained.

Theorem 2.2. Let $f$ be given by (1) be in the class $S L M_{\lambda, t, \Sigma}(\tilde{p}(z))$. Then

$$
\left|a_{2}\right| \leq \frac{|\tau|}{\sqrt{4(1+\lambda)^{2}(1-t)^{2}+\left\{3(1+2 \lambda)\left(2-t-t^{2}\right)-8(1+\lambda)^{2}\left(2-3 t+t^{2}\right)\right\} \tau}}
$$

and

$\left|a_{3}\right|<\frac{|t| 4(1+\lambda)^{2}\left((1-t)^{2}-2\left(2-3 t+t^{2}\right) \tau\right)}{3(1+2 \lambda)\left(2-t-t^{2}\right) 4(1+\lambda)^{2}(1-t)^{2}+\left(3(1+2 \lambda)\left(2-t-t^{2}\right)-8(1+\lambda)^{2}\left(2-3 t+t^{2}\right)\right) t}$

Proof. Let $f \in S L M_{\lambda, t, \Sigma}(\tilde{p}(z))$ and $g=f^{-1}$ Considering (13) and (14), we have

$$
\frac{(1-t)\left(\lambda z^{3} f^{\prime \prime \prime}(z)+(1+2 \lambda) z^{2} f^{\prime \prime}(z)+z f^{\prime}(z)\right)}{\lambda z^{2}\left(f^{\prime \prime}(z)-t^{2} f^{\prime \prime}(t z)\right)+z\left(f^{\prime}(z)-t f^{\prime}(t z)\right)}=\tilde{p}(u(z))
$$

and

$$
\frac{(1-t)\left(\lambda w^{3} g^{\prime \prime \prime}(w)+(1+2 \lambda) w^{2} g^{\prime \prime}(w)+w g(w)\right)}{\lambda w^{2}\left(g^{\prime \prime}(w)-t^{2} g^{\prime \prime}(t w)\right)+w\left(g^{\prime}(w)-t^{\prime}(t w)\right)}=\tilde{p}(v(w))
$$

Where

$$
\tau=\frac{(1-\sqrt{5})}{2} \approx-0618
$$
where $z, w \in \Omega$ and $g$

is given by (2).

Since

$(1-t)\left(\lambda z^{3} f^{\prime \prime \prime}(z)+(1+2 \lambda) z^{2} f^{\prime \prime}(z)+z f^{\prime}(z)\right)$

$\lambda z^{2}\left(f^{\prime \prime}(z)-t^{2} f^{\prime \prime}(t z)\right)+z\left(f^{\prime}(z)-t f^{\prime}(t z)\right)$

$=1+2(1+\lambda)(1-t) a_{2} z+\left(3(1+2 \lambda)\left(2-t-t^{2}\right) a_{3}-4\left(1-t^{2}\right)(1+\lambda)^{2} a_{2}^{2}\right) z^{2}+\ldots$

and

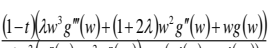

$\left.2 w^{2} g^{\prime \prime}(w)-t^{2} g^{\prime \prime}(t w)\right)+w\left(g^{\prime}(w)-t g^{\prime}(t w)\right)$

$=1-2\left(1+\lambda(1-t) a_{2} w+\left(\left(6(1+2 \lambda)\left(2-t-t^{2}\right)-4\left(1-t^{2}\right)(1+\lambda)^{2}\right)_{2}^{2}\right)-3(1+2 \lambda)\left(2-t-t^{2}\right) a_{3}\right) w^{2}+\ldots .$. Thus we have

$1+2(1+\lambda)(1-t) a_{2} z+\left(3(1+2 \lambda)\left(2-t-t^{2}\right) a_{3}-4\left(1-t^{2}\right)(1+\lambda)^{2} a_{2}^{2} z^{2}+\ldots\right.$

$=1+\frac{\tilde{p}_{1} c_{1} z}{2}+\left\{\frac{1}{2}\left(c_{2}-\frac{c_{1}^{2}}{2}\right) \tilde{p}_{1}+\frac{c_{1}^{2}}{4} \tilde{p}_{2}\right\} z^{2}+$

$\left\{\frac{1}{2}\left(c_{3}-c_{1} c_{2}+\frac{c_{1}^{2}}{4}\right) \tilde{p}_{1}+\frac{1}{2} c_{1}\left(c_{2}-\frac{c_{1}^{2}}{2}\right) \tilde{p}_{2}+\frac{c_{1}^{3}}{8} \tilde{p}_{3}\right\} z^{3}+\ldots \ldots$.

and

$1-2\left(1+\lambda(1-t) a_{2} w+\left(\left(6(1+2 \lambda)\left(2-t-t^{2}\right)-4\left(1-t^{2}\right)(1+\lambda)^{2}\right) a_{2}^{2}\right)-3(1+2 \lambda)\left(2-t-t^{2}\right) a_{3}\right) w^{2}+\ldots$ 
Fekete - Szego Problem for Sakaguchi kind of Functions Related to Shell - like Curves Connected with Fibonacci numbers

$$
\begin{aligned}
& =1+\frac{\tilde{p}_{1} d_{1} w}{2}+\left\{\frac{1}{2}\left(d_{2}-\frac{d_{1}^{2}}{2}\right) \tilde{p}_{1}+\frac{d_{1}^{2}}{4} \tilde{p}_{2}\right\} w^{2}+ \\
& \left\{\frac{1}{2}\left(d_{3}-d_{1} d_{2}+\frac{d_{1}^{2}}{4}\right) \tilde{p}_{1}+\frac{1}{2} d_{1}\left(d_{2}-\frac{d_{1}^{2}}{2}\right) \tilde{p}_{2}+\frac{d_{1}^{3}}{8} \tilde{p}_{3}\right\} w^{3}+\ldots \ldots . .
\end{aligned}
$$

It follows from (19) and (20) that

$$
2(1+\lambda)(1-t) a_{2}=\frac{c_{1} \tau}{2}
$$

$$
3(1+2 \lambda)\left(2-t-t^{2}\right) a_{3}-4\left(1-t^{2}\right)(1+\lambda)^{2} a_{2}^{2}=\frac{1}{2}\left(c_{2}-\frac{c_{1}^{2}}{2}\right) \tau+\frac{c_{1}^{2}}{4} 3 \tau^{2},
$$

and

$$
-2(1+\lambda)(1-t) a_{2}=\frac{d_{1} \tau}{2}
$$

$\left(2\left(3(1+2 \lambda)\left(2-t-t^{2}\right)-2\left(1-t^{2}\right)(1+\lambda)^{2}\right) a_{2}^{2}\right)-3(1+2 \lambda)\left(2-t-t^{2}\right) a_{3}$ $=\frac{1}{2}\left(d_{2}-\frac{d_{1}^{2}}{2}\right) \tau+\frac{d_{1}^{2}}{4} 3 \tau^{2}$

From (21) and (23), we have

$$
c_{1}=-d_{1}
$$

and

$$
8 a_{2}^{2}=\frac{\left(c_{1}^{2}+d_{1}^{2}\right) \tau^{2}}{4(1+\lambda)^{2}(1-t)^{2}}
$$

Now, by summing (22) and (24), we obtain $\left(6(1+2 \lambda)\left(2-t-t^{2}\right)-8(1+\lambda)^{2}\left(1-t^{2}\right)\right) a_{2}^{2}=\frac{1}{2}\left(c_{2}+d_{2}\right) \tau-\frac{1}{4}\left(c_{1}^{2}+d_{1}^{2}\right) \tau+\frac{3}{4}\left(c_{1}^{2}+d_{1}^{2}\right) \tau^{2}$

By putting (26) in (27), we have

$2\left(4(1+\lambda)^{2}(1-t)^{2}+\left(3(1+2 \lambda)\left(2-t-t^{2}\right)-8(1+\lambda)^{2}\left(2-3 t+t^{2}\right)\right) \tau\right) a_{2}^{2}=\frac{1}{2}\left(c_{2}+d_{2}\right) \tau^{2}$

Therefore, using Lemma1.4, we obtain

$$
\left|a_{2}\right| \leq \frac{|\tau|}{\sqrt{4(1+\lambda)^{2}(1-t)^{2}+\left(3(1+2 \lambda)\left(2-t-t^{2}\right)-8(1+\lambda)^{2}\left(2-3 t+t^{2}\right)\right) \tau}}
$$

Now, so as to find the bound on $\left|a_{3}\right|$, let's subtract from (22) and (24). So, we find $6(1+2 \lambda)\left(2-t-t^{2}\right) a_{3}-6(1+2 \lambda)\left(2-t-t^{2}\right) a_{2}^{2}=\frac{1}{2}\left(c_{2}-d_{2}\right)$.

Hence, we get

$6(1+2 \lambda)\left(2-t-t^{2}\right)\left|a_{3}\right| \leq 2|\tau|+6(1+2 \lambda)\left(2-t-t^{2}\right)\left|a_{2}^{2}\right|$

Then, in view of (29), we obtain

$\left|a_{3}\right| \leq \frac{|t|(1+\lambda)^{2}\left((1-t)^{2}-2\left(2-3 t+t^{2}\right) \tau\right.}{3(1+2 \lambda)\left(2-t-t^{2}\right)\left(4(1+\lambda)^{2}(1-t)^{2}+\left(3(1+2 \lambda)\left(2-t-t^{2}\right)-8(1+\lambda)^{2}\left(2-3 t+t^{2}\right)\right) t\right.}$

If we can take the parameter $\lambda=0$ in the above theorem, we have the following the initial Taylor coefficients $\left|a_{2}\right|$ and $\left|a_{3}\right|$ for the function class $K L_{\Sigma, t}(\tilde{p}(z))$.

Corollary 2.3. Let $f$ given by (1) be in the class $K S L_{\Sigma}(\tilde{p}(z))$. Then

$$
\left|a_{2}\right| \leq \frac{|\tau|}{\sqrt{4(1-t)^{2}+\left(3\left(2-t-t^{2}\right)-8\left(2-3 t+t^{2}\right)\right) \tau}}
$$

And

$$
\left|a_{3}\right| \leq \frac{|\tau| 4\left((1-t)^{2}-2\left(2-3 t+t^{2}\right) \tau\right)}{3\left(2-t-t^{2}\right)\left\{4(1-t)^{2}+\left(3\left(2-t-t^{2}\right)-8\left(2-3 t+t^{2}\right)\right) \tau\right\}}
$$

Taking $t=0$, we get the following corollary which is obtained by [9].

Corollary 2.4. Let $f$ given by (1) be in the class $K S L_{\Sigma}(\tilde{p}(z))$. Then

$$
\left|a_{2}\right| \leq \frac{|\tau|}{\sqrt{4-10 \tau}}
$$

and

$$
\left|a_{3}\right| \leq \frac{|\tau|(1-4 \tau)}{3(2-5 \tau)}
$$

\section{FEKETE- SZEgo INEQUALITIES FOR THE FUNCTION$$
\text { CLASS } S L M_{\lambda, t, \Sigma}(\tilde{p}(z))
$$

Fekete and Szego [6] introduced the generalized functional $\left|a_{3}-\mu a_{2}^{2}\right|$, where $\mu_{\text {is some real number. Due to }}$ Zaprawa [15], in the following theorem we determine the Fekete - Szego functional for $f \in S L M_{\lambda, t, \Sigma}(\tilde{p}(z))$.

Theorem 3.1. let $f$ given by (1) be in the class $S L M_{\lambda, t, \Sigma}(\tilde{p}(z))$ and $\mu \in \mathfrak{R}$. Then we have

$$
\left|a_{3}-\mu a_{2}^{2}\right| \leq\left\{\begin{array}{c}
\frac{|\tau|}{3(1+2 \lambda)\left(2-t-t^{2}\right)},|\mu-1| \leq \frac{A_{1}}{A_{2}} \\
\frac{4(1-\mu) \tau^{2}}{2 A_{1}},|\mu-1| \geq \frac{A_{1}}{A_{2}}
\end{array}\right.
$$

Where

$A_{1}=8(1+\lambda)^{2}(1-t)^{2}+\left(6(1+2 \lambda)\left(2-t-t^{2}\right)-16(1+\lambda)^{2}\left(2-3 t+t^{2}\right)\right) \tau$ and

$$
A_{2}=6(1+2 \lambda)\left(2-t-t^{2}\right) \tau \mid
$$

Proof . From (28) and (30) the following equation is obtained

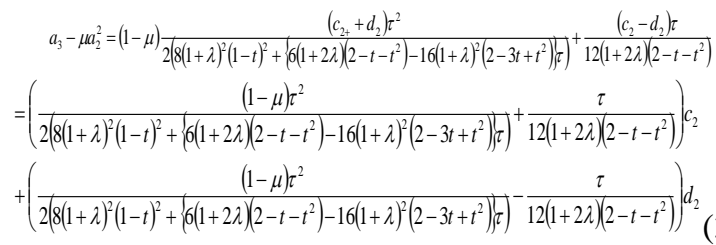

Therefore

$a_{3}-\mu a_{2}^{2}=\left(h(\mu)+\frac{\tau}{12(1+2 \lambda)\left(2-t-t^{2}\right)}\right) c_{2}+\left(h(\mu)-\frac{\tau}{12(1+2 \lambda)\left(2-t-t^{2}\right)}\right) d_{2}$

where

$$
h(\mu)=\frac{(1-\mu) \tau^{2}}{2\left(8(1+\lambda)^{2}(1-t)^{2}+\left\{6(1+2 \lambda)\left(2-t-t^{2}\right)-16(1+\lambda)^{2}\left(2-3 t+t^{2}\right)\right\} \tau\right)}
$$

Then, by taking modulus of (38), we conclude that 


$$
\left|a_{3}-\mu a_{2}^{2}\right| \leq\left\{\begin{array}{c}
\frac{|\tau|}{3(1+2 \lambda)\left(2-t-t^{2}\right)}, 0 \leq \frac{|\tau|}{12(1+2 \lambda)\left(2-t-t^{2}\right)} \\
4|h(\mu),| h(\mu) \mid \geq \frac{|\tau|}{12(1+2 \lambda)\left(2-t-t^{2}\right)}
\end{array}\right.
$$

Taking $\mu=1$, the following corollary is obtained.

Corollary 3.2. If $f \in S L M_{\lambda, t, \Sigma}(\tilde{p}(z))$, then

$$
\left|a_{3}-a_{2}^{2}\right| \leq \frac{|\tau|}{3(1+2 \lambda)\left(2-t-t^{2}\right)}
$$

If we take the parameter $\lambda=0$ in the above theorem, we have the following the Fekete - Szego inequality for the function class $K S L_{\Sigma}(\tilde{p}(z))$.

Corollary 3.3. Let $f$ given by (1) be in the class $K S L_{\Sigma}(\tilde{p}(z))_{\text {and }} \mu \in \mathfrak{R}$

Then we have

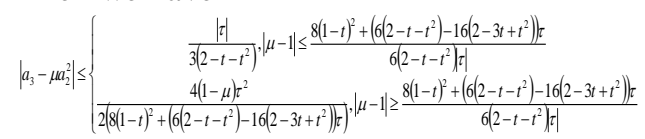

Taking $t=0$ we get the following corollary which is obtained by [9].

Corollary 3.4. Let $f$ given by (1) be in the class $K S L_{\Sigma}(\tilde{p}(z))$ and $\mu \in \mathfrak{R}$. Then we have

$$
\left|a_{3}-\mu a_{2}^{2}\right| \leq\left\{\begin{array}{c}
\frac{|\tau|}{6},|\mu-1| \leq \frac{2-5 \tau}{3|\tau|} \\
\frac{(1-\mu) \tau^{2}}{2(2-5 \tau)},|\mu-1| \geq \frac{2-5 \tau}{3|\tau|}
\end{array}\right.
$$

\section{CONCLUSION}

It is attempted to introduce and investigate new subclasses of Sakaguchi kind of functions related to shell - likes curves connected with Fibonacci numbers. Furthermore, the estimates of first two coefficients of functions in these classes are obtained. Fekete - Szego inequalities for these function classes are also determined.

\section{REFERENCES}

1. D.A. Brannan, J. Clunie and W.E. Kirwan, Coefficient estimates for a class of star-like functions, Canad. J. Math., 22 (1970), 476-485.

2. D.A. Brannan and T.S. Taha, On some classes of bi-univalent functions, Stud. Univ. Babes-Bolyai Math., 31(2) (1986), 70-77.

3. P.L. Duren, Univalent Functions. In: Grundlehren der Mathematishcen Wissenschaften, Band 259, New York, Berlin, Heidelberg and Tokyo, Springer-Verlag, 1983.

4. J. Dziok, R.K. Raina and J. Sokol, Certain results for a class of convex functions related to a shell-like curve connected with Fibonacci numbers, Comp. Math. Appl., 61 (2011), 2605-2613.

5. J. Dziok, R.K. Raina and J. Sokol, On $\alpha$-convex functions related to a shell-like curve connected with Fibonacci numbers, Appl. Math. Comp., 218 (2011), 996-1002.

6. M. Fekete and G. Szego, Eine Bemerkung uber ungerade schlichte Functionen, J. London Math. Soc., 8 (1933), 85-89.

7. S.S. Miller and P.T. Mocanu, Differential Subordinations Theory and Applications, Series of Monographs and Text Books in Pure and Applied Mathematics, 225, Marcel Dekker, New York (2000).
8. M. Lewin, On a coefficient problem for bi-univalent functions, Proc. Amer. Math. Soc., 18 (1967), 63-68.

9. H. Ozlem Guney, G. Murusundaramoorth and J. Sokol, Subclasses of biunivalent functions related to shell-like curves connected with Fibonacci numbers, Acta Univ. Sapientiae mathematica, 10(1) (2018), 70-84.

10. Ch. Pommerenke, Univalent functions, Math. Math, Lehrbucher, Vandenheock and Ruprecht, Gottingen, (1975).

11. R.K. Raina and J. Sokol, Fekete-Szego problem for some starlike functions related to shell-like curves, Math. Slovaca, 66 (2016), 135-140.

12. J. Sokol, On starlike functions connected with Fibonacci numbers, Folia Scient. Univ. Tech. Resoviensis, 175 (1999), 111-116.

13. H.M. Srivastava, A.K. Mishra and P. Gochhayat, Certain subclasses of analytic and bi-univalent functions, Appl. Math. Lett., 23(10) (2010), 1188-1192. Q.-H. Xu, Y.-C. Gui and H.M. Srivastava, Coefficient estimates for a certain subclass of analytic and bi-univalent functions, Appl. Math. Lett., 25 (2012), 990-994.

14. X.-F. Li and A.-P. Wang, Two new subclasses of bi-univalent functions, International Mathematical Forum, 7(30) (2012), 1495-1504.

15. P. Zaprawa, On the Fekete-Szego problem for classes of bi-univalent functions, Bull. Belg. Math. Soc. Simon Stevin, 21(1) (2014), 169-178.

\section{AUTHORS PROFILE}

P.Lokesh Research Scholar, Department of Mathematics, Bharathiar University.

B.Srutha keerthi Mathematics Division, School of Advanced Sciences, VIT University Chennai Campus, Vandallur kellambakkam Road, Chennai 600127, India 